\title{
Disc Monopole Antenna with Ring Patches and Meandered Feeder for Radio Frequency Energy Harvesting
}

\author{
Nada N. Tawfeeq ${ }^{1}$, Kaydar M. Quboa ${ }^{2, *}$ \\ ${ }^{1}$ Department of Electrical Engineering, University of Tikrit, College of Engineering, Salah Al-Din, Iraq \\ ${ }^{2}$ Department of Electrical Engineering, University of Mosul, College of Engineering, Mosul, Iraq \\ *Corresponding author: kaydar_quboa@yahoo.com
}

Received February 27, 2014; Revised March 17, 2014; Accepted March 22, 2014

\begin{abstract}
In this paper, a new wideband antenna with meandered feed line is presented which can be used in radio frequency (RF) energy harvesting. The proposed antenna is designed on 36 by $56.5 \mathrm{~mm}^{2}$ FR4 board with thickness of $1.6 \mathrm{~mm}$ and dielectric permittivity constant and tangent loss of 4.3 and 0.025 , respectively. It consists of circular disc antenna, two ring patches and three rectangular slots on the ground plane as well as a meandered feed line. Although any RF signal could be useful from energy harvesting point of view, the proposed antenna shows a wideband frequency range from 1.59 to $6.62 \mathrm{GHz}$ and $7.44 \mathrm{GHz}$ to $8 \mathrm{GHz}$ in reference to $-10 \mathrm{~dB}$ return loss or better for wideband applications.
\end{abstract}

Keywords: wideband, monopole antenna, meandering

Cite This Article: Nada N. Tawfeeq, and Kaydar M. Quboa, "Disc Monopole Antenna with Ring Patches and Meandered Feeder for Radio Frequency Energy Harvesting." American Journal of Electrical and Electronic Engineering, vol. 2, no. 3 (2014): 67-71. doi: 10.12691/ajeee-2-3-2.

\section{Introduction}

All the time, there are wireless transmissions of power at different frequencies, and most of the transmitted energy is wasted. Based on this fact, the concept of radio frequency energy harvesting has been introduced. By energy harvesting, any source of energy at a certain level could be harness to be used for recharging a mobile phones battery; operating battery-less devices and many other applications.

Within the last decade, the wireless transmission technique has begun to open, and adopted in many used portable devices. As the usage of these hand held electronic devices is increasing, the demands for longer battery life are also increasing. Available radio frequency (RF) energy in the environment or areas close to transmission sources provides an opportunity to harvest that energy using frequencies up to ultra wide band (UWB) $[1,2]$.

There has been research on UWB, which extends from $3.1 \mathrm{GHz}$ to $10.6 \mathrm{GHz}$ according to Federal Communication Commission (FCC), especially for wireless communications [3].

Research on wide bands to cover lower frequencies in the Megahertz range to high frequencies in the Gigahertz range is continuously being carried out [4,5]. The size of the antenna, which is very important element in wireless application, is different depends on the targeted wideband. It is small for high frequency band and large for low frequency band [6].
The proposed antenna is designed to be used in RF energy harvesting to cover several used bands such as, GSM 1800, GSM 1900, WLAN-2.4, and WLAN-5.2, or any other standard bands.

The proposed antenna is based on a circular disc monopole antenna because of its radiatingr patch shape to obtain small size; simple structure and wide bandwidth [7]. The proposed antenna uses a combination of different techniques such as creating new paths to current by adding two ring patches on the sides and adding slots on both the radiating and ground planes to increase the bandwidth by making irregular surface currents [8]; cutting a step in upper half of the radiating patch [9] and meandering the feeding line to lengthen the path of the current [10]. Using all these techniques will give additional features to the proposed antenna.

In this paper, two antennas are designed and simulated, using Computer Simulation Technology (CST) Microwave Studio package, to be used for wireless energy harvesting application. The first proposed antenna is a disc monopole with straight feed line (DMSFL), and the second proposed antenna is a disc monopole with meandered feed line (DMMFL).

Both antennas are designed on $W=36 \mathrm{~mm}$ by $L=56.5$ mm FR4 substrate with thickness of $1.6 \mathrm{~mm}$ and relative dielectric constant of 4.3 .

\section{DMSFL Antenna Design}

The DMSFL antenna is a disc monopole with ring patches and straight feed line. The radiating patch of this antenna is shown in Figure 1. 


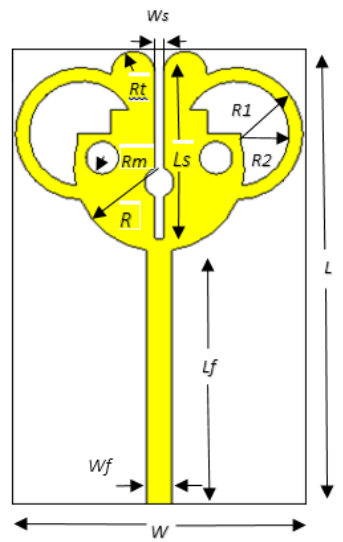

Figure 1. The radiating patch of DMSFL antenna

All parameters shown in Figure 1 are optimized for better return loss and wider bandwidth. It consists of a disc patch of radius $R=10.5 \mathrm{~mm}$; two ring patches of outer radius $R 1=8.2 \mathrm{~mm}$ and inner radius $R 2=6.8 \mathrm{~mm}$; a straight feed line of dimensions $W f=3 \mathrm{~mm}$ and $L f=31.5$ $\mathrm{mm}$; a rectangular slot of size $W s=1 \mathrm{~mm}$ by $L s=24 \mathrm{~mm}$ and a circular slot of radius $R m=1.8 \mathrm{~mm}$ to improve the matching at low frequencies; and additional two circular patches on the top of the rings with radius $R t=2.7 \mathrm{~mm}$ are added.

The ground patch of DMSFL antenna is shown in Figure 2.

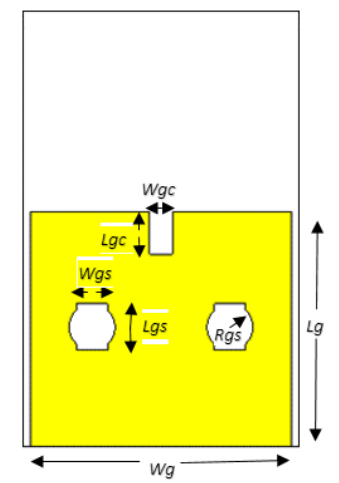

Figure 2. The ground patch of DMSFL antenna

Again all parameters shown in Figure 2 are optimized for better RL and wider bandwidth. The ground patch is found to be of size $W g=34 \mathrm{~mm}$ by $L g=30.5 \mathrm{~mm}$ which has two symmetric slots, each is a combination of a circular sector of radius $R g s=3 \mathrm{~mm}$ and a rectangle of dimensions $W g s=4 \mathrm{~mm}$ by $L g s=6 \mathrm{~mm}$. In addition, a third rectangular slot of dimensions $W g c=3.2 \mathrm{~mm}$ by $\mathrm{Lgc}$ $=6 \mathrm{~mm}$ is extracted from the upper edge of the ground patch for better performance.

\section{DMSFL Antenna Results and Discussion}

The DMSFL antenna is simulated using CST Microwave Studio. The simulated return loss (RL) is shown in Figure 3.

Assuming -10dB return loss level as an acceptable RL, corresponding to a voltage standing wave ratio (VSWR) of 2, Figure 3 shows an interesting band of frequencies starting from 1.71 to $6.11 \mathrm{GHz}$.

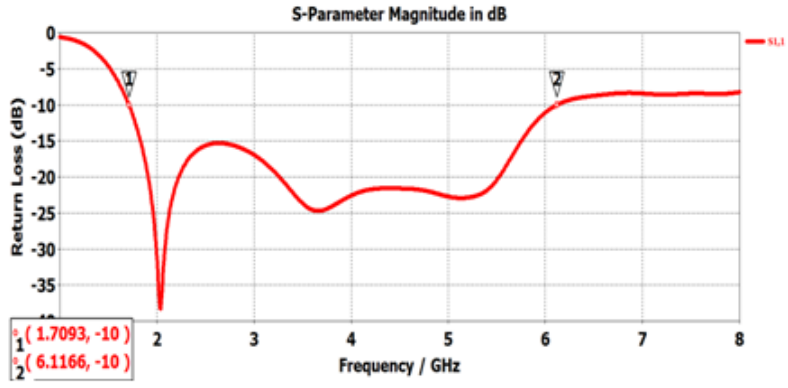

Figure 3. The simulated return loss of DMSFL antenna

The impedance of DMSFL antenna is simulated and the result is shown in Figure 4.

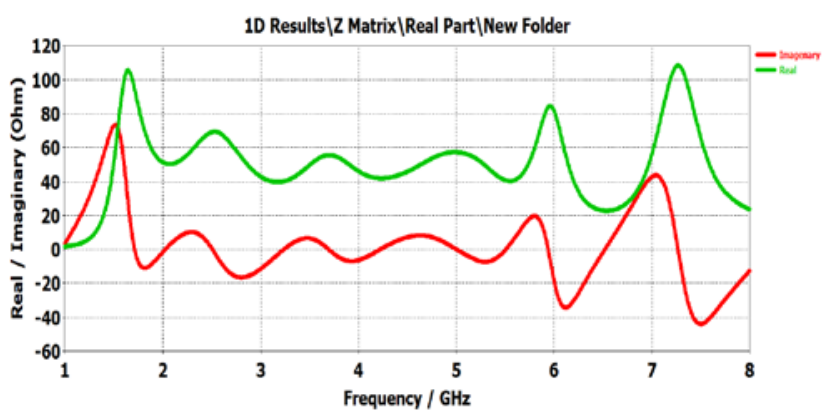

Figure 4. The real and imaginary parts of the $\mathrm{Z}$ matrix of DMSFL antenna

It could be noted from Figure 4 that the real part of DMSFL antenna impedance is varying between $50 \pm 10 \Omega$ in the frequency range from 1.71 to $6.11 \mathrm{GHz}$ and the imaginary part is varying between $0 \pm 10 \Omega$ in the same frequency range indicating an acceptable matching with $50 \Omega$.

\section{DMMFL Antenna Design}

The DMMFL antenna is a disc monopole with ring patches with meandered feed line, as shown in Figure 5, to lengthen the current path of the feeder for better performance.

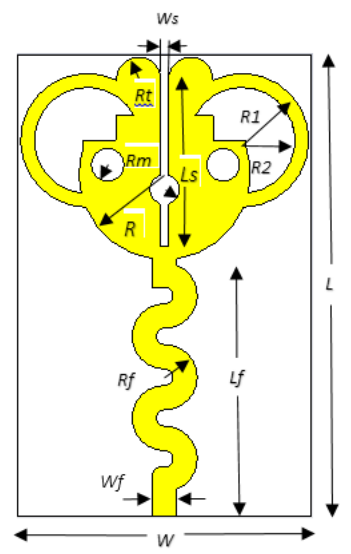

Figure 5. The radiating patch of DMMFL antenna

All parameters shown in Figure 5 for the radiating patch are fixed to the same optimized values as those of DMSFL antenna except the feed line which is meandered by curved paths successively through all the line with radius of radius of $R f=4 \mathrm{~mm}$.

The ground patch of DMMFL antenna is the same as that of DMSFL antenna shown in Figure 2 except the 
dimension of $\mathrm{Lgs}$ which is optimized to a value of $8 \mathrm{~mm}$ as shown in Figure 6.

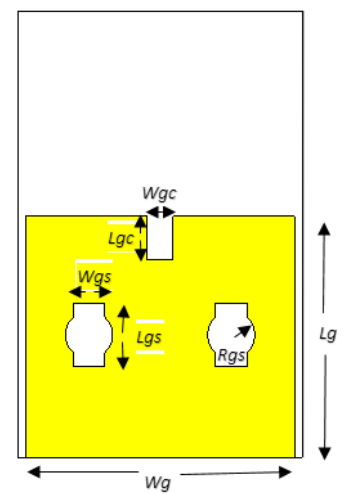

Figure 6. The ground patch of DMSFL antenna

\section{DMMFL Antenna Results and Discussion}

The DMMFL antenna is simulated using CST Microwave Studio. The simulated return loss (RL) is shown in Figure 7. It shows two interesting bands of frequencies starting from 1.59 to $6.62 \mathrm{GHz}$ and from 7.43GHz to nearly $8 \mathrm{GHz}$ at $-10 \mathrm{~dB}$ RL reference level.

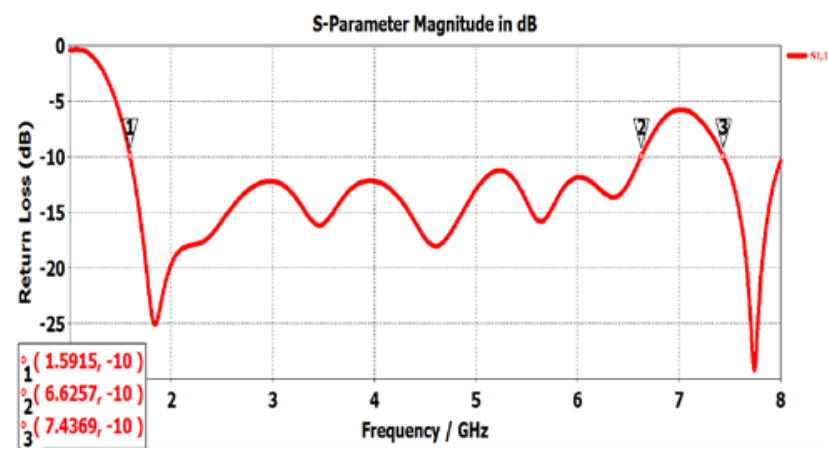

Figure 7. The simulated return loss of DMMFL antenna

As can be seen from Figure 3 \& Figure 7, there has been improvement in the bandwidth of DMMFL antenna by adding $120 \mathrm{MHz}$ to the lower cutoff frequency of DMSFL antenna, and adding another $51 \mathrm{MHz}$ to the upper cutoff frequency limit. Besides that, an additional band of frequencies staring from $7.4 \mathrm{GHz}$ to nearly $8 \mathrm{GHz}$ in reference to $-10 \mathrm{~dB}$ RL level is obtained.

The impedance of DMMFL antenna is simulated and the result is shown in Figure 8.

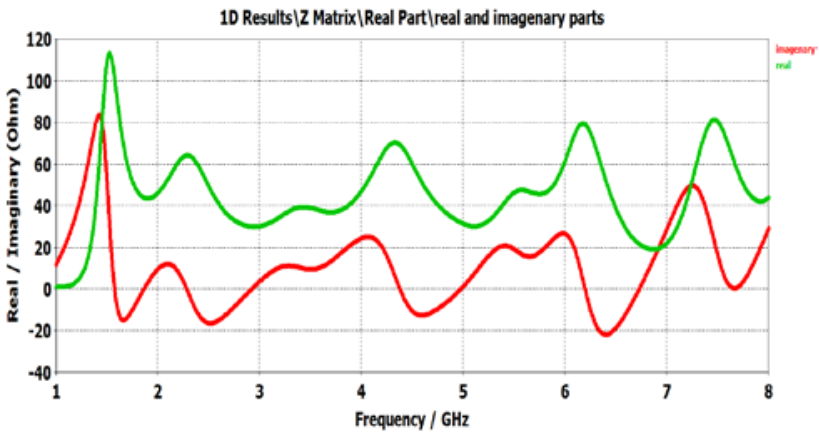

Figure 8. The simulated real and imaginary parts of the $\mathrm{Z}$ matrix of DMMFL antenna
It is clear from Figure 8 that by using meandering technique an inductive behavior at 4.06, 5.4, and $6 \mathrm{GHz}$ where it showed capacitive behavior in the straight technique. At $4.58 \mathrm{GHz}$, the inductive behavior in the straight feed line becomes capacitive with the meandering feed line.

Because DMMFL antenna shows better performance compared to DMSFL antenna, further investigations are carried out.

The gain of DMMFL antenna is simulated as a function of frequency as shown in Figure 9, which shows a gain above $1.4 \mathrm{~dB}$ at frequency above $5 \mathrm{GHz}$ and maximum gain of $2.5 \mathrm{~dB}$ at $2.4 \mathrm{GHz}$.The impedance matching between the antenna and the port is shown in Figure 10 using Smith chart, where the antenna impedance becomes closer to the line impedance of $49.95 \Omega$.

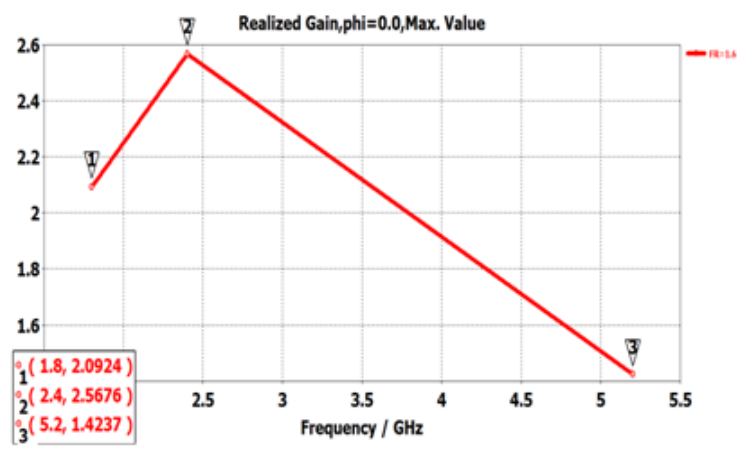

Figure 9. Realized gain of the proposed DMMFL antenna

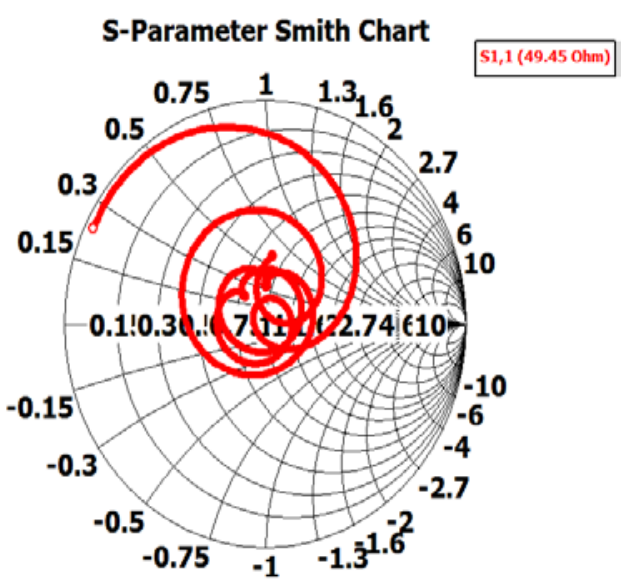

Figure 10. Smith Chart of the proposed DMMFL antenna

The value of VSWR reaches maximum of 1.755 at 5.25 $\mathrm{GHz}$ and doesn't exceed 2 through the bandwidth as shown in Figure 11.

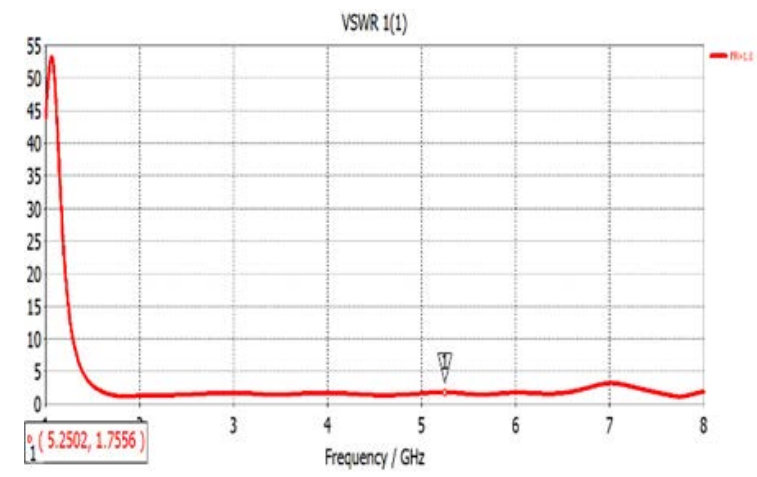

Figure 11. Simulated VSWR of DMMFL antenna 
Figure 12 shows the efficiency of the DMMFL antenna. It can be seen that a maximum efficiency of $91.56 \%$ at $1.8 \mathrm{GHz}$ and as the frequency increases the efficiency decreases reaching $72.31 \%$ at $5.2 \mathrm{GHz}$.

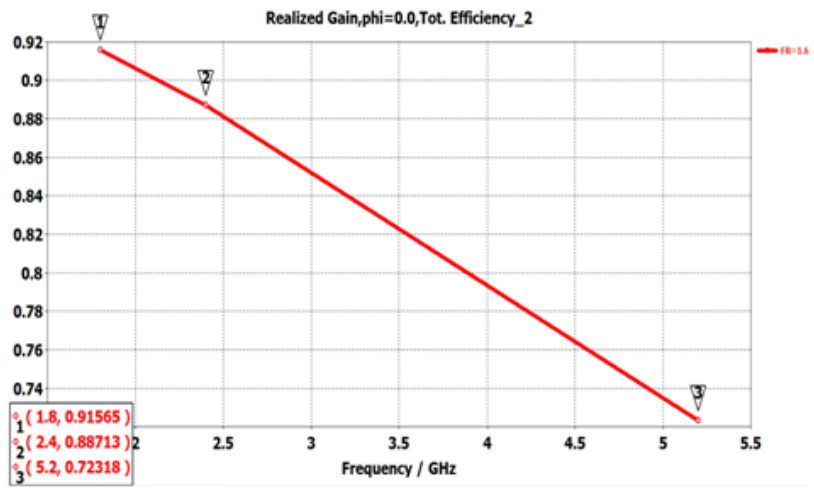

Figure 12. Total efficiency of the proposed DMMFL antenna

The current density distribution for the proposed DMMFL antenna at three different frequencies of 1.8, 2.4 and $5.2 \mathrm{GHz}$ are shown in Figure 13. These frequencies are chosen because they present the most common used standards in wireless communication.

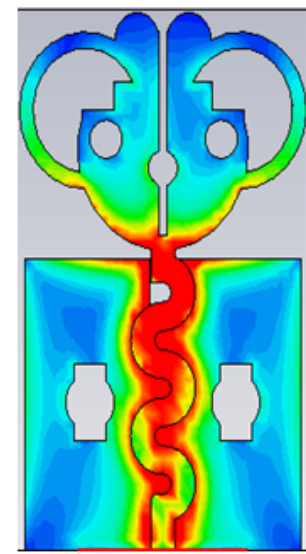

(a)

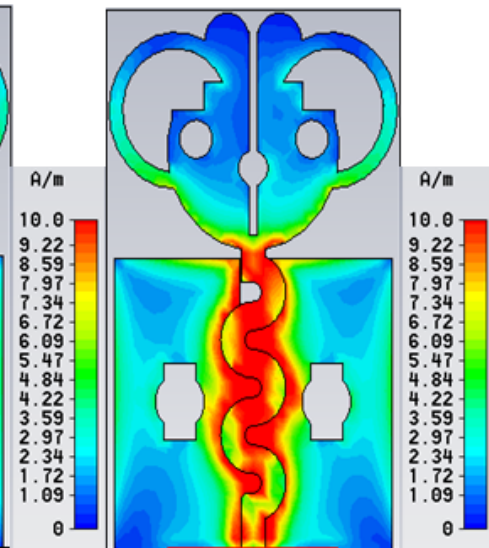

(b)

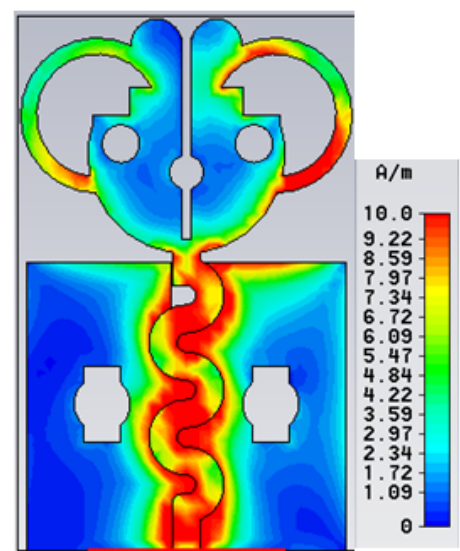

(c)

Figure 13. Current distribution for DMMFL antenna at: (a) $1.8 \mathrm{GHz}$, (b) $2.4 \mathrm{GHz}$, (c) $5.2 \mathrm{GHz}$

The blue, green, and red colors shown in Figure 13 denote low, moderate, and high current density respectively. The electric currents are concentrated along the feeding line, while it is concentrated at the beginning of the patch at $1.8 \mathrm{GHz}$. On the other hand, the current density is less at $2.4 \mathrm{GHz}$ and starts to spread through the ground patch. At $5.2 \mathrm{GHz}$, the current is less concentrated at the beginning of the radiating patch and moves to the ring patches of the antenna in asymmetrical manner.

Figure 14, shows the radiation far-field plot in 3D for the two frequencies of $2.4 \mathrm{GHz}$ and $5.2 \mathrm{GHz}$. Comparing between them, it can be noticed that at $2.4 \mathrm{GHz}$ the radiation is focused around the feed line on both the front and back and less on the sides of the antenna, while at 5.2 $\mathrm{GHz}$ the radiation is concentrated around the feed line on one side and the rings of the antenna, which agrees with the current distribution shown in Figure 13.

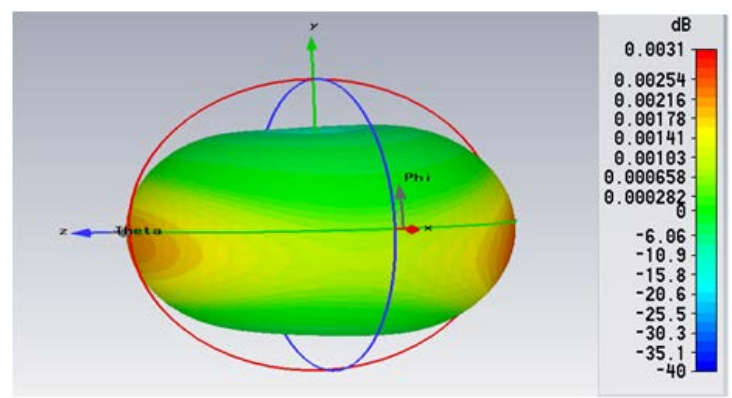

(a)

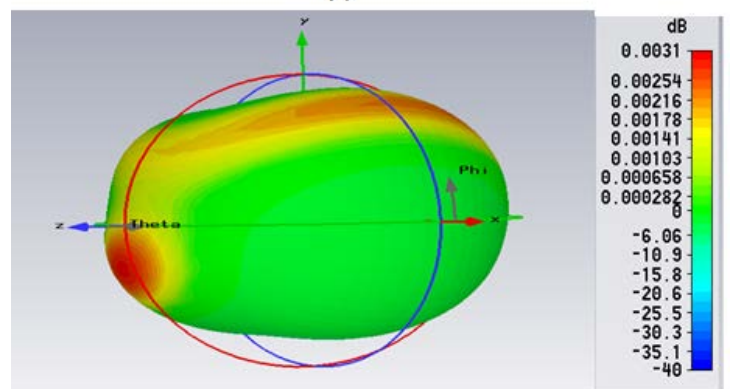

(b)

Figure 14. Far-field radiation pattern in 3D at: (a) $2.4 \mathrm{GHz}$, and (b) 5.2 $\mathrm{GHz}$

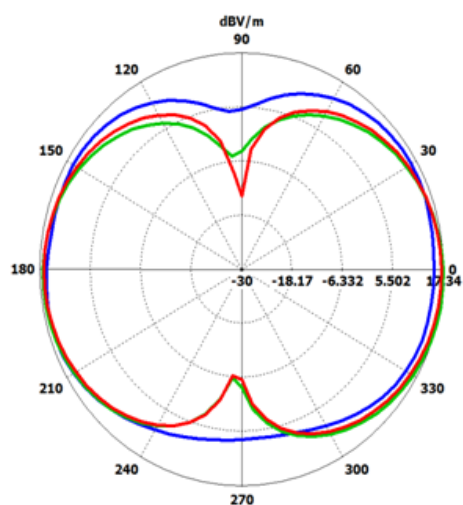

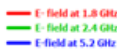

(a)

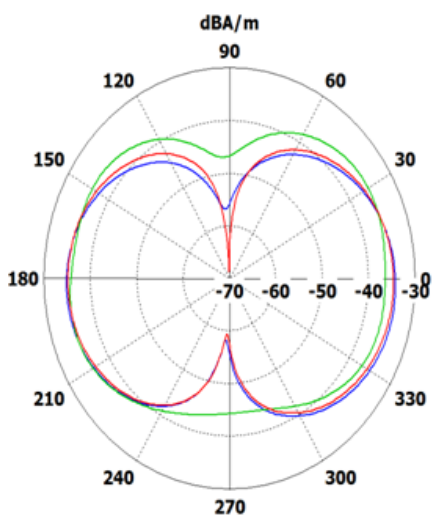

(b)

Figure 15. Simulated radiation pattern for DMMFL antenna at 1.8, 2.4, and $5.2 \mathrm{GHz}$ in the: (a) E-plane, and (b) H-plane 
Figure 15 shows the simulated E-plane and H-plane at 1.8, 2.4, and 5.2 GHz. It can be noticed that the radiation pattern is nearly Omni-directional as frequency increases.

The size, bandwidth, and gain of the proposed disc monopole with ring patches and meandered feed line (DMMFL) antenna are compared with those given in [6] and [8] as given in Table 1.

Table 1. Comparison Between DMMFL Antenna with Those in [6] and [8]

\begin{tabular}{|c|c|c|c|c|}
\hline & Size & Bandwidth & Gain (dB) & Year \\
\hline Ref. [8] & 30 by $51 \mathrm{~mm}^{2}$ & 1.98 to $6.46 \mathrm{GHz}$ & 3 & 2010 \\
\hline Ref. [6] & 120 by150 $\mathrm{mm}^{2}$ & 0.77 to $11.23 \mathrm{GHz}$ & 4.3 & 2012 \\
\hline DMMFL & 36 by $56.5 \mathrm{~mm}^{2}$ & 1.59 to $6.62 \mathrm{GHz}$ & 2.565 & This paper \\
\hline
\end{tabular}

As can be seen from Table 1, the proposed DMMFL antenna has almost the same area as that in [8] but it has a larger bandwidth and that increment in dimensions is necessary to reach the above lower frequency limit of the bandwidth, unlike [6] where it has an area 8 times larger than that of the proposed antenna to achieve double the bandwidth of our proposed antenna. As for the gain, the DMMFL antenna has a gain of $2.58 \mathrm{~dB}$ which is less than that given in [6] and [8].

\section{Conclusion}

The circular disc monopole antenna with two ring patches fed by meandered feed line is presented in this paper. With the overall adjustment and optimization of the geometrical parameters, the proposed antenna can operate between 1.59 to $6.62 \mathrm{GHz}$ and at $7.44 \mathrm{GHz}$ to $8 \mathrm{GHz}$ for wide band applications. By using different techniques, the antenna shows small size, good matching, and acceptable gain to be integrated in portable devices for radio frequency energy harvesting.

\section{References}

[1] Khoury, P, A power-efficient radio frequency energy-harvesting circuit, M.Sc. thesis, University of Ottawa, 2013. Available at : http://hdl.handle.net/10393/23627.

[2] Cesar, M. G, Ultra-Wideband (UWB) Rectenna Design for Electromagnetic Energy Harvesting, M.Sc. thesis, Universitat Politecnica de Cataluny, 2010. Available at : http://hdl.handle.net/2099.1/10835

[3] Gary, B., Ed., A Summery of FCC Rules for Ultra Wide Communication: High Frequency Electronics, Summit Technical Media, 2005.

[4] Peter, T., Cheung, S. W., Rahim, S. K., and Tgarek, A. R, “A grounded CPW transperant UWB antenna for UHF and microwave frequency application,” Electromagnetics Research Symposium Proceedings, Taipei, March 25-28, 2013.

[5] Peyrot-Solis, M. A., Tirado-Mendez, J. A., Galvan-Tejada, G. M., and Jardon-Aguilar, H, "Implementation of an Ultra-Wide Band Planar Monopole Antenna for Operation from $650 \mathrm{MHz}$ to 20 GHz," in 10th WSEAS International Conference on Communictions, World Scientific and Engineering Academy and Society (WSEAS), 479-483.

[6] Gomez-Nunez, E., Jardon-Aguilar, H., Tirado-Mendez, J. A., and Flores-Leal, R, "Ultra-Wide Band Slotted Disc Antenna Compatable with Cognitive Radio Applications," Electromagnetic research letters, 34, 53-63, 2012.

[7] Mohammed, A., Ed., Ultra-wide band antenna and design,in Ultra wide band-current status and future, InTech, 2012. [E-book]. Available at: http://www.intechopen.com/books/ultra-widebandcurrent-status-and-future-trends/ultra-wideband-antenna-anddesign.

[8] Aghda, M. R. and Kamarudin, M. R, "UTM-Logo Wide Band Printed Monopole Antenna Surrounded with Circular Ring Patch," Electromagnetic research C, 15, 157-164, 2010.

[9] Ahmed, K., Ed., UWB antennas for wireless applications: Microstrip antennas with recent applications, InTech, 2013, [Ebook].

Available at: http://www.intechopen.com/books/advancement-inmicrostrip-antennas-with-recent-applications/uwb-antennas-forwireless-applications.

[10] Li, D., Shu-Fang, L., Ka-Leung, L.,and Quan, X. "Vertical Meandering Approach for Antenna Size Reduction,” International Journal of Antennas and Propagation, 2012, 5, 2012. 